\title{
Better functional outcomes for ORIF in tibial eminence fracture treatment: a national comparative multicentric study of ORIF vs ARIF
}

\author{
Régis Pailhé ${ }^{1,9} \cdot$ Thomas Bauer $^{2} \cdot$ Xavier Flecher $^{3,4} \cdot$ Nicolas Bonnevialle $^{5} \cdot$ Xavier Roussignol $^{6}$. \\ Dominique Saragaglia ${ }^{1} \cdot$ Matthieu Ehlinger ${ }^{7} \cdot$ The SOFCOT $^{8}$
}

\begin{abstract}
Purpose Tibial eminence fractures can occur in adults and are equivalent to an acute ACL rupture. The purpose of this retrospective study was to compare the therapeutic outcomes of ARIF versus ORIF in tibial eminence fractures. Our hypothesis was that ARIF does not offer better results.

Methods A retrospective national multicentric study was conducted in five university hospitals between 2010 and 2015 . A total of 65 consecutive patients were included. 33 patients were treated with ARIF and 32 with ORIF. Clinical functional outcomes were assessed using the Lysholm score and IKDC score. Radiographic findings were recorded, and a statistical analysis carried out.

Results IKDC score at the mean last follow-up of $68.8 \pm 11.8$ months was significantly higher in the ORIF group with a mean difference of 20.2 points $\pm 8.9(p=0.028)$. There were early osteoarthritis findings in 12 patients $(18.4 \%)$. At last follow-up, 7 patients $(10.7 \%)$ presented complications.

Conclusion In this retrospective multicentric study, better functional outcomes were observed in the ORIF group. This difference needs to be carefully interpreted as many confounding factors exist. In terms of complications, the results for both ORIF and ARIF are similar at midterm follow-up. ORIF should remain gold standard for tibial eminence fracture treatment. Level of evidence III.
\end{abstract}

Keywords Tibial eminence fracture · ARIF · ORIF

\section{INTRODUCTION}

Tibial eminence fractures are significantly less frequent than intraligamentous tears of the ACL [6]. Most tibial eminence fractures occur in children and adolescents (age 8-14 years), but these injuries can also be seen in adults

Régis Pailhé

rpailhe@chu-grenoble.fr

1 Department of Orthopaedic Surgery and Sport Traumatology, Grenoble South Teaching Hospital, Avenue de Kimberley, BP 338, 38434 Échirolles Cedex, France

2 Hôpitaux Universitaires Paris Ile-de-France Ouest, Assistance Publique-Hôpitaux de Paris, 92100 Boulogne-Billancourt, France

3 Institute for Locomotion, Aix-Marseille University, Marseille, France

4 Department of Orthopedics and Traumatology, St Marguerite Hospital, Marseille, France and are equivalent to an acute rupture of the ACL [2]. Concomitant injury to the menisci, articular cartilage, and collateral ligaments can also be seen, and are more common in adults [1,2]. Tibial eminence fractures are commonly classified according to the criteria established by Meyers and McKeever. Surgical treatment must be proposed for

5 Institut Locomoteur, Hôpital Pierre-Paul Riquet, Place du Docteur Baylac, 31059 Toulouse, France

6 Department of Orthopaedics Surgery and Traumatology, Rouen University Hospital, Rouen, France

7 Service de Chirurgie Orthopédique Et de Traumatologie, Hôpital de Hautepierre, Hôpitaux Universitaires de Strasbourg, 1 Avenue Molière, 67098 Strasbourg Cedex, France

8 SOFCOT, 56 Rue Boissonade, 75014 Paris Cedex, France

9 Service de Chirurgie Orthopédique, Hôpital Sud Grenoble, Grenoble Cedex, France 
types 2, 3 and 4 [9]. An open approach was traditionally used, however, surgically treated fractures are now increasingly managed arthroscopically $[1,8,14]$. Successful results have been reported with both methods [2]. Nonetheless, it is unclear whether arthroscopic reduction and internal fixation (ARIF) offer additional benefits when compared with conventional open reduction and internal fixation (ORIF). To our knowledge, this is the first study to compare the therapeutic outcomes of ARIF versus ORIF in tibial eminence fractures. The purpose of this retrospective study was to explore whether ARIF is equivalent to ORIF. The hypothesis was that ARIF does not offer better results.

\section{Materials and methods}

A retrospective national multicentric study was conducted in five university hospitals between 2010 and 2015. All patients operated on for an isolated tibial eminence fracture were included. Patients with an associated tibial plateau fracture, patients under 14 years old and those with follow-up of less than 3 years were excluded.

Sixty-five patients fulfilled these criteria and were included. There were 36 males and 29 females with a mean age of 31.6 years \pm 13 (range 14-59 years). There were 33 patients treated with ARIF, and 32 treated with ORIF. All patients were injured in either sport activities or road traffic accidents. All patients underwent standard knee radiographic examination. In $65 \%$ of cases, preoperative planning consisted of computed tomography scanning with three-dimensional reconstruction. An MRI was carried out before surgery in only $20 \%$ of cases. Fracture configuration and associated lesions are presented in Table 1 . The mean delay before surgery was 3.3 days \pm 3.4 (range: $1-15$ days). The procedure was performed by a junior surgeon (having graduated less than 3 years previously) in $60 \%$ of cases.

Patients treated with ORIF were operated on using minimally invasive medial approach in all cases. Fixation was carried out with isolated screwing in $40 \%$ of cases, button plate suture in $40 \%$ of cases and an EndoButton in 20\% of cases-in the ARIF group only. Post-operative instructions were immobilization in a knee brace for the first 45 days with full weightbearing. From 45 days post-surgery,

Table 1 PrEoperative descriptive analysis

\begin{tabular}{llll}
\hline Criteria & ORIF & ARIF & $p$ value \\
\hline Age of patient (years) & $31.5(14-59)$ & $28.0(17-46)$ & \\
Duration of surgery (min) & $49.3(15-90)$ & $76.8(35-135)$ & $<0.05$ \\
Associated lesions & & & \\
Meniscal & $n=2(6.3 \%)$ & $N=4(12.1 \%)$ & $<0.05$ \\
Chondral & $n=3(9.4 \%)$ & $N=6(18.2 \%)$ & $<0.05$ \\
\hline
\end{tabular}

unrestricted active movement and quadriceps strengthening were authorized.

Patients were evaluated by independent observers in a clinical setting or contacted by video chat when they were unable to come to clinic. A letter was sent out to perform and collect radiographs at last follow-up.

Participation in sports and recreational activities, level of proficiency, and number of hours per week during the year prior the accident, and at the time of follow-up, were collected. If patients did not recover to their prior level of sporting proficiency or activity they were asked to define the reason. The Lysholm score [15] and IKDC score [4] were used to assess the clinical functional outcomes.

Radiographic findings were recorded. Radiological parameters studied were: quality of fracture reduction (anatomic reduction, displacement $>2 \mathrm{~mm}$, displacement $>5 \mathrm{~mm}$ ), consolidation, secondary displacement, material failure, osteoarthritis according to Kellgren-Lawrence classification [7]. Complications and additional surgeries on the affected knee, such as implant removal, pseudarthrosis, knee arthroscopic surgery, joint replacement were reported.

Institutional (SOFCOT) review board approval was obtained (IRB ID 2017-SF-069).

\section{Statistical analysis}

Statistical analysis was carried out using EXCEL® (Microsoft Inc., Redmond, Wash.) and SPSS software ${ }^{\circledR}$ (SPSS Inc., Chicago, Ill). Gaussian distribution of continuous variables was verified using the Shapiro-Wilk test. The equality of variances corresponding to the condition of homoscedasticity required for the use of parametric tests was verified using the Levene test. Descriptive data analysis was performed using student $t$ test. Preoperative and follow-up data were compared using paired Student $t$ tests. The effect size of 10 points for Lysholm score, significance level of 0.05 and $90 \%$ power of the study on paired Student $t$ tests suggested a sample size of 15 patients in each group.

\section{Results}

\section{Descriptive analysis}

The mean follow-up period was $68.8 \pm 11.8$ months (range 36-100). There were no statistical differences between both groups in terms of age, sex, BMI and type of fracture. Surgery was performed with ORIF in $77 \%$ of patients by senior surgeon while ARIF was realized in $77 \%$ of patients by junior surgeon. The mean surgical time was $65 \pm 15.3 \mathrm{~min}$ (range: 15-135). The mean duration of ARIF was $30 \mathrm{~min}$ longer than ORIF $(p<0.05)$. More associated lesions were found with ARIF procedure and meniscal sutures or 
resections were carried out in 4 cases $(12.1 \%)$ while none of the ORIF patients had a meniscal treatment (Table 1). No chondral repair was performed in either group (Fig 1).

\section{Functional outcomes}

All functional outcomes are reported in Table 2. The mean time of return to sports activities was $20.7 \pm 18.9$ weeks (range: 10-100). At last follow-up, the average Lysholm score was $84.6 \pm 19$ points (range: $59-100$ ). The average IKDC score was $74.5 \pm 31$ points (range: $54-97$ ) and $81 \%$ of patients were satisfied or very satisfied with the surgical management.

IKDC score at the latest follow-up was significantly higher in the ORIF group with a mean difference of $20.2 \pm 8.9$ points $(p=0.028)$. Active extension was also significantly better in the ORIF group with a mean difference of $3.1 \pm 1.2^{\circ}(p=0.010)$.

\section{Radiological analysis}

Immediate postoperative radiographs demonstrated anatomic reduction was achieved in $87 \%$ of cases and was maintained throughout the follow-up period. Reduction was considered anatomical for 26 patients $(81.2 \%)$ in the ORIF group and 28 patients $(84.8 \%$ ) in the ARIF group (n.s). No malunion nor secondary displacement was observed. Further radiographic analysis demonstrated that osteoarthritis developed in 12 patients (18.4\%). Nonetheless, only one patient presented a Kellgren-Lawrence grade superior to grade 2 (grade $1 ; n=9$, grade $2 ; n=2$, grade $4 ; n=1$ ).

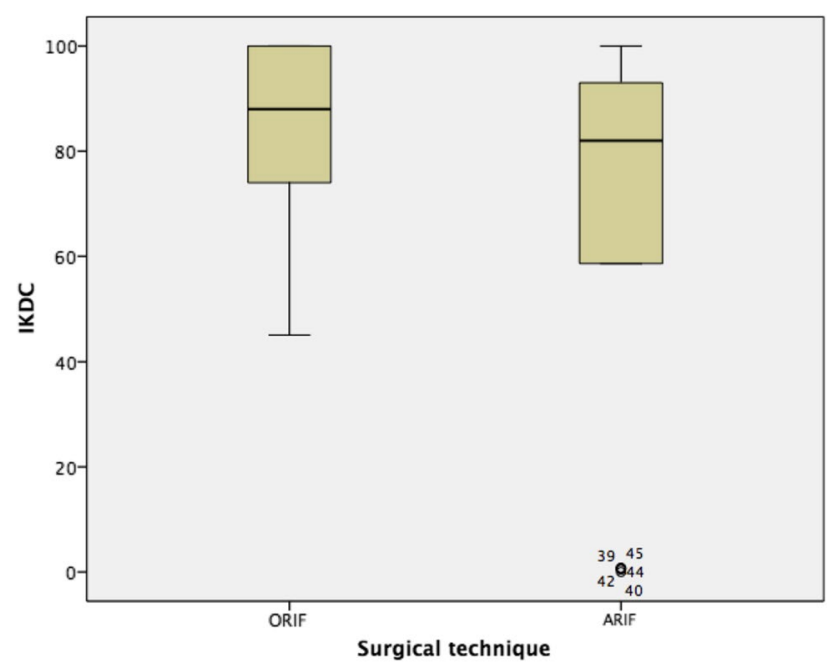

Fig. 1 Box plot of IKDC scores at last follow-up (outliers patients are represented by circles with their number id)
Table 2 Comparison of postoperative outcomes at last follow-up

\begin{tabular}{lccl}
\hline & \multicolumn{1}{l}{ ORIF } & \multicolumn{1}{l}{ ARIF } & $p$ value \\
\hline $\begin{array}{l}\text { Time of return to } \\
\text { sports (weeks) }\end{array}$ & $20.4 \pm 11.4$ & $20.9 \pm 22.8$ & n.s \\
Lysholm score & $87.2 \pm 16.3$ & $81.9 \pm 21.3$ & n.s \\
IKDC score & $85.4 \pm 15.1$ & $65.2 \pm 38.3$ & $p=0.028$ \\
Range of motions & & & \\
$\quad$ Flexion & $129.2 \pm 10.0$ & $122.6 \pm 29.7$ & n.s \\
$\quad$ Extension & $1.1 \pm 2.6$ & $-2 \pm 5.2$ & $p=0.010$ \\
\hline
\end{tabular}

\section{Complications}

At last follow-up, seven patients (10.7\%) presented complications. Two patients reported instability and five patients reported stiffness. Details of complications and revisions are presented in Table 3. No statistical differences were observed between the two groups. Nevertheless, the only complication in the arthroscopic group was knee stiffness.

\section{Discussion}

This study aimed to compare the therapeutic outcomes of ARIF versus ORIF in tibial eminence fractures. ORIF demonstrated better functional outcomes while the rate of complications was similar in both groups, disproving our hypothesis.

Recently, in a systematic review, Bogunovic et al. [2] provided a synthesis of the literature regarding the outcome of non-surgical and surgically treated displaced tibial eminence fractures. Despite several limitations, a clear trend could be seen in this systematic assessment of outcomes following nonoperatively and operatively treated tibial eminence fractures. Nonoperative treatment of displaced fractures resulted in increasing rates of clinical and subjective instability and subsequent need for ACL reconstruction. While both suture and screw fixation restored knee function [14].

Regarding comparison of arthroscopic versus open fixation, there have been no comparative studies published.

Table 3 Complications and revisions details

\begin{tabular}{lll}
\hline & ORIF $=9.4 \%$ & ARIF $=12.4 \%$ \\
\hline Complications & Instability: $n=2$ & Knee stiffness: $n=4$ \\
& Knee stiffness: $n=1$ & \\
Revisions & ACL reconstruction: $n=2$ & Arthrolysis: $n=4$ \\
& Mobilisation under anesthesia: & \\
& $n=1$ \\
& Patellar tendon debridement: & \\
& $n=1$ & \\
\hline
\end{tabular}


Many series of cases report excellent functional outcomes for both ORIF and ARIF [1-3, 14, 17]. We did not find any evidence in literature to support the hypothesis that ORIF might have better functional outcomes. Published data on the arthroscopic management and functional outcomes of ARIF are less abundant for adults than for adolescents [2, 12]. Bone suture has been reported to be superior to screw fixation, as the latter is linked to an increased incidence of repeated surgery for hardware removal [2, 14]. In this study no difference was observed with regard to the fixation device used. The two most common complications after ARIF are residual laxity (associated with non-operative management or technical errors during surgery) and post-operative stiffness. The rate of these complications has been reported to be between 13 and 38\% in the literature [2], while it was just $3 \%$ (two patients) for instability and of $8 \%$ (five patients) for stiffness in this series. Even if no significant difference between groups was observed in this study, knee stiffness was the only type of complication reported in the ARIF group.

Indeed, postoperative stiffness of the knee is the most common complication observed in many series, due to arthrofibrosis or mechanical impingement of displaced bony fragment [11, 17]. Thaunat et al. [16], believe that unlike traditional ACL reconstruction which is performed after a few weeks, once any inflammation has subsided, in avulsion injuries, surgery is performed on an inflamed knee followed by immobilization. Recently, however, many authors have reported minimal incidence of arthrofibrosis after arthroscopic rigid fixation and early mobilization within 2-4 weeks, suggesting that early mobilization can reduce the rate of arthrofibrosis and improve the outcome [10, 11, 17].

Eminential fractures are associated with significant risk for concomitant knee injuries including meniscal tear, bone contusion, and chondral injury [13]. Recently, Green et al. [5], recommended a systematic MRI-based assessment as it provides specific, quantitative criteria for classifying fractures according to fragment displacement and tissue entrapment. In this study, more meniscal and chondral lesions were observed with patients treated arthroscopically. Consequently, meniscal sutures or resections were performed in $11.8 \%$ of cases in the ARIF group, while none of the ORIF patients required meniscal treatment. Even if we assume that meniscal lesions were probably underestimated in the ORIF group, functional outcomes and complication rates were not affected at last follow-up. There was no difference between the two groups in terms of osteoarthritis and lesions were always lower than Kellgren-Lawrence classification grade 2.

Considering reported outcomes, the main clinical relevance of this study is that ORIF should remain the gold standard for eminential fractures.

This study did, however, have limitations. First, it was a multicentric retrospective study combining different operators and surgical techniques. Second, a majority of ARIFs were carried out by junior surgeons, leading to a possible a bias when comparing functional outcomes between the groups. This bias is mitigated by the similar rate of complications in both groups.

\section{Conclusion}

In this multicentric, retrospective study, we observed better functional outcomes in the ORIF group. This difference needs to be interpreted carefully as many confounding factors exist. In terms of complications, ORIF and ARIF results are similar at midterm follow-up.

Author contributions All authors did acquisition and analysis of data. All authors have read and approved the final submitted manuscript.

Funding No external funding was used.

\section{Compliance with ethical standards}

Conflict of interest Authors declare that they have no conflict of interest related to this study.

Ethical approval Each author certifies that his or her institution approved the human protocol for this investigation and that all investigations were conducted in conformity with ethical principles of research.

Informed consent Informed consent was obtained for all subjects who participated in the study.

\section{References}

1. JH Ahn JC Yoo 2005 Clinical outcome of arthroscopic reduction and suture for displaced acute and chronic tibial spine fractures Knee Surg Sports Traumatol Arthrosc 13116121

2. L Bogunovic M Tarabichi D Harris R Wright 2015 Treatment of tibial eminence fractures: a systematic review J Knee Surg 28255 262

3. N Bonin L Jeunet L Obert D Dejour 2007 Adult tibial eminence fracture fixation: arthroscopic procedure using K-wire folded fixation Knee Surg Sports Traumatol Arthrosc 15857862

4. NJ Collins D Misra DT Felson KM Crossley EM Roos 2011 Measures of knee function: International Knee Documentation Committee (IKDC) Subjective Knee Evaluation Form, Knee Injury and Osteoarthritis Outcome Score (KOOS), Knee Injury and Osteoarthritis Outcome Score Physical Function Short Form (KOOS-PS), Knee Ou Arthritis Care Res (Hoboken) 63 S208 S228

5. D Green M Tuca E Luderowski E Gausden C Goodbody G Konin 2019 A new, MRI-based classification system for tibial spine fractures changes clinical treatment recommendations when compared to Myers and Mckeever Knee Surg Sports Traumatol Arthrosc 27 8692 
6. AM Joseph CL Collins NM Henke EE Yard SK Fields RD Comstock 2013 A multisport epidemiologic comparison of anterior cruciate ligament injuries in high school athletics J Athl Train 48 810817

7. JH Kellgren JS Lawrence 1957 Radiological assessment of osteoarthrosis Ann Rheum Dis 16494502

8. P Loriaut P-E Moreau P Loriaut P Boyer 2017 Arthroscopic treatment of displaced tibial eminence fractures using a suspensory fixation Indian J Orthop 51187191

9. MH Meyers FM McKeever 1970 Fracture of the intercondylar eminence of the tibia J Bone Jt Surg Am 5216771684

10. FR Noyes S Berrios-Torres SD Barber-Westin TP Heckmann 2000 Prevention of permanent arthrofibrosis after anterior cruciate ligament reconstruction alone or combined with associated procedures: a prospective study in 443 knees Knee Surg Sports Traumatol Arthrosc 8196206

11. SN Parikh D Myer EA Eismann 2014 Prevention of arthrofibrosis after arthroscopic screw fixation of tibial spine fracture in children and adolescents Orthopedics 37 e58 65

12. R Rajanish M Jaseel C Murugan CM Kumaran 2018 Arthroscopic tibial spine fracture fixation: novel techniques J Orthop 15372 374
13. JT Rhodes PC Cannamela AI Cruz M Mayo AC Styhl CG Richmond TJ Ganley KG Shea 2018 Incidence of meniscal entrapment and associated knee injuries in tibial spine avulsions J Pediatr Orthop 38 e 38 e 42

14. W Sang L Zhu J Ma H Lu Y Yu 2012 A comparative study of two methods for treating type III tibial eminence avulsion fracture in adults Knee Surg Sports Traumatol Arthrosc 2015601564

15. Y Tegner J Lysholm 1985 Rating systems in the evaluation of knee ligament injuries Clin Orthop Relat Res 1984349

16. M Thaunat NC Barbosa R Gardon S Tuteja R Chatellard J-M Fayard B Sonnery-Cottet 2016 Prevalence of knee stiffness after arthroscopic bone suture fixation of tibial spine avulsion fractures in adults Orthop Traumatol Surg Res 102625629

17. KL Vander Have TJ Ganley MS Kocher CT Price JA Herrera-Soto 2010 Arthrofibrosis after surgical fixation of tibial eminence fractures in children and adolescents Am J Sports Med 38298301 